\title{
The Association Between Mental Health, Chronic Disease, and Retired Status Among Korean Elderly: A Cross-Sectional Study
}

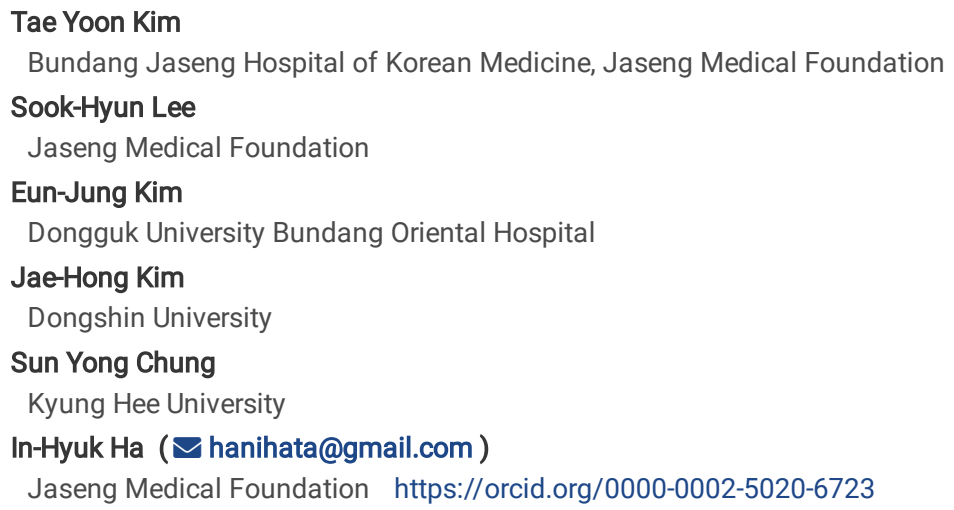




\begin{abstract}
Background Retired status has strong associations with mental health factors and involves gender disparities. However, while retirement is known to affect the mental health of the elderly, large-scale studies examining the impact on mental health by gender are scarce.

Methods This cross-sectional study examined the data of 1,664 participants aged over 65 years old who completed the economic status and mental health sections of the 6-7th Korean National Health and Nutrition Examination Surveys (2013-2017). We adjusted for covariates (health behavior, sociodemographic factors) in regression analysis for complex sampling design to calculate regression coefficients and $95 \%$ confidence intervals of the link between retired status and mental health. We also assessed which covariates of chronic disease, health behavior, and sociodemographic factors weakened the correlation between retired status and mental health.
\end{abstract}

Results Retired status in Korean populations has persisted to negatively impact the incidence of depressive symptoms and thoughts of suicide, even after adjusting for confounding variables. Regarding mental health, women were affected by retired status to a greater extent than men. Among covariates, health behaviors and sociodemographic factors were most affected by retired status. Women were more affected by household income, and men were more affected by drinking behaviors.

Conclusions These results imply that chronic diseases and psychological factors should be managed delicately among the retired elderly, and that there may be gender differences in retirement adaptation.

\title{
Background
}

Increases in the life expectancy and aging of the population are leading to longer periods of old age, and the resulting higher numbers of people in the aged population have renewed the focus on studies that explore health-related factors in old age. The importance of employment for the maintenance of health cannot be overlooked, as workplaces act as key contexts for the formation of the ego and the identity of the self [1].

Retirement, signaling the loss of job functions for the elderly, constitutes the process of stepping away from a career on which the individual has worked for his/her entire life; adjustments that the individual makes during this period of rapid change can be reasonably inferred to have a significant impact on his/her health status.

Hugo et al. reported that while retirement was associated with reduced levels of physical and mental fatigue, it was also associated with a higher prevalence of respiratory diseases, diabetes, coronary artery diseases, and stroke with increasing age [2]. Furthermore, from the perspective of mental health, previous studies have reported that retirement among the elderly is related to reduced adaptability [3], increased stress [4], and worsened mental health issues such as depression [5].

A cross-sectional study among Australians reported that retired status is related to depression and anxiety. However, the study was limited in that it failed to consider the influence of depression and anxiety on chronic diseases, given the correlation between chronic disease and retired status [6]. Furthermore, a largescale longitudinal study by Song [7] that utilized data from the National Survey of Families and Households reported that retired state was related to the mental health of the elderly in the United States; however, it only included a covariate of depression symptoms among many mental health-related variables.

The present study was designed to overcome the structural limitations of previous studies, and to understand the gender differences regarding the influence of retired status on the sub-items of mental health among the elderly Korean population. The purpose of this study was to understand whether retired status, covariates of mental health, and prevalence of chronic disease are related, and whether the covariates of mental health show gender-specific patterns using the cross-sectional data from the 6-7th Korean National Health and Nutrition Examination Surveys (KNHANES VI-VII).

\section{Methods}

Study Population And Sampling

This study was carried out using the KNHANES VI-VII data collected between January 2013 and December 2017, from which a representative sample of the Korean population was obtained using a rolling sampling design involving stratified, multi-stage, and probability cluster sampling. This study was organized by the Ministry of Health and Welfare in Korea, and the survey items were organized into three domains of health, nutrition, and health check-up. Details can be found in "The 6-7th KNHANES Sample Design," which can be accessed on the KNHANES website at https://knhanes.cdc.go.kr/knhanes/index.do. The KNHANES annual report, user manual and guidelines, and raw data are available on request. Out of 39,225 potential respondents, 10,479 individuals (26.7\%) participated in the 6-7th KNHANES surveys. We excluded 7,812 respondents aged below 65 years old, 1,095 respondents who did not 


\section{provide answers regarding their status of economic activity, and 113 respondents who were classified as unemployed for reasons other than retirement. Ultimately, responses from 1,664 respondents were analyzed. After modifying for propensity score matching (PSM), data from 475 employed people and 95 retired people were utilized in the analysis (Fig. 1.)}

\section{Definitions Of Retired Status}

We defined retired status among the respondents of KNHANES VI-VII as being aged older than 65 years and being unemployed due to age of retirement/retired status.

\section{Mental Health}

Perception of stress was classified into three categories: high (as indicated by a response of "very high" or "high"), low (as indicated by a response of "low") and almost none (as indicated by a response of "under almost no stress"). Psychiatric diagnosis of depression and experience of suicidal ideation were recorded by responses of "yes" or "no"; answer selections were based on whether the respondent had received a psychiatric diagnosis of depression and whether they had considered suicide, respectively.

\section{Chronic Disease}

The presence of chronic diseases including hypertension, hyperlipidemia, osteoarthritis, rheumatoid arthritis, diabetes, osteoporosis, pulmonary tuberculosis, and asthma was noted; respondents answered "yes" if they had been diagnosed by a physician and "no" if they had not.

\section{Covariates}

Demographic characteristics such as gender and socioeconomic, lifestyle-related, and health-related characteristics were evaluated as covariates (age, sex, place of residence, education, marital status, household income, BMl, smoking, drinking, level of physical activity, and subjective perceived health). Sex was classified as "male" and "female," and age was treated as a continuous variable. Place of residence was categorized into "rural areas" and "urban areas." Education was classified into three categories of "elementary school graduate," "middle school graduate," and "high school graduate or higher." Marital status was categorized into "residing with spouse" and "not residing with spouse." Household income level was subject to equalization (dividing the monthly average household income by the number of members in a household), and then divided into two categories based on monthly average household income. BMI $(\mathrm{kg} / \mathrm{m} 2)$ was classified according to the World Health Organization's criteria and then reclassified into three categories: "underweight," "normal weight," and "overweight or obese." Smoking status was divided into "non-smoker," "ex-smoker," and "current smoker." Drinking habits were divided into four levels: (a) Nondrinkers, defined as not having consumed alcohol in the past year; (b) Drinking once or less per month; (c) Drinking twice or more per month but less than 3 times a week; and (d) drinking more than four times a week. Regular walking was divided into "yes" or "no," depending on whether the respondent had walked more than 10 minutes for at least one day in the past week. Subjective health was classified into four categories: "very good" (as indicated by responses of "very good"), "good" (as indicated by responses of "good"), "moderate" (as indicated by responses of "moderate"), "poor" (as indicated by responses of "poor"), and "fair" (as indicated by any other responses).

\section{Statistical analysis}

KNHANES is a national study that applies both stratified cluster sampling and weighted values. Among various analysis methods of complex sampling designs, this study used stratification, clustering, and weighted variables for complex sampling design factors to analyze the data. All analyses were conducted using SAS version.9.4. (SAS Institute, Cary, North Carolina, USA), and were considered to be statistically significant if $p<0.05$. Means and standard deviations are reported for continuous variables, and means and percentages (\%) are reported for categorical variables. This study also utilized PSM to reduce the baseline differences of comorbidities. Thereafter, PSM was conducted using nearest neighbor matching without replacements for 1:5 matching. We used the "pscore" and "psmatch2" modules in SAS to calculate propensity scores. Differences in characteristics due to retired status were evaluated using the RaoScott chi-square test or a t-test. We also used a complex sample design regression analysis modified for covariates to calculate regression coefficients and $95 \%$ confidence intervals $(\mathrm{Cl})$, evaluating the sizes of correlation between mental health and retired status. Age, sex, and other covariates were included in all covariate models as individual covariates, covariate blocks, and full models, and used to calculate the regression coefficients on retired status.

\section{Ethics Statement}

KNHANES VI-VII were conducted by the Korea Center for Disease Control and Prevention (KCDC). All survey protocols were approved by the Institutional Review Board (IRB) of the KCDC (approval numbers: 2013-07CON-03-4C and 2013-12EXP-03-5C). In 2015, 2016, and 2017, they were carried out without reviews as per the IRB of the Centers for Disease Control (for state research for public welfare). Informed consent was obtained from all participants when the surveys were conducted. Original data are publicly available for free on the KNHANES website (http://knhanes.cdc.go.kr) for purposes such as academic 
research. IRB approval was not required for the present study because it did not involve any sensitive information and accessed only publicly available data from KNHANES (JASENG IRB File No. 2020-03-002).

\section{Results}

\section{Participant Characteristics Reflecting Retired Status (before/after Propensity Matching)}

Table 1 summarizes the clinical characteristics of the sample based on PSM. According to standardized bias (\%), the clinical characteristics of the retired and non-retired groups were biased prior to PSM. Although we were not able to calibrate for minor differences, these biases were reduced significantly after $1: 5$ PSM. After PSM, a total of 570 participants were categorized according to sex (Table 2). Among men $(n=351)$, the majority of characteristics including the covariates of mental health did not differ significantly, aside from residence $(p=0.0171)$ between groups. Among women $(n=219)$, there were significant differences only in the household income level $(p=0.0097)$; however, the general patterns of characteristics were similar to those among men (Table 2). 
Table 1

Characteristics of Korean elderly $\geq 65$ years participating in KNHANES VI -VII (2013-2017), before and after PSMa.

\begin{tabular}{|c|c|c|c|c|c|c|}
\hline \multirow[t]{4}{*}{ Variable } & \multicolumn{3}{|c|}{ Before propensity score matching } & \multicolumn{3}{|c|}{ After propensity score matching } \\
\hline & Retired & Employed & P-value & Retired & Employed & P-value \\
\hline & Status $^{b}$ & Status $^{c}$ & & Status & Status & \\
\hline & $(n=297)$ & $(n=1367)$ & & $(n=95)$ & $(n=475)$ & \\
\hline \multicolumn{7}{|l|}{ Demographic factors } \\
\hline \multicolumn{7}{|l|}{ Gender, n (\%) } \\
\hline Male & $237(78.56)$ & 766(55.81) & $<0.0001$ & $59(55.87)$ & 292(59.12) & 0.6311 \\
\hline Female & $60(21.44)$ & $601(44.19)$ & & $36(44.13)$ & $183(40.88)$ & \\
\hline \multicolumn{7}{|l|}{ Age $(y), n(\%)$ or mean \pm SE } \\
\hline $65-69$ & 104(35.98) & $639(46.74)$ & 0.0001 & $42(39.72)$ & $211(43.70)$ & 0.8167 \\
\hline $70-74$ & $83(26.52)$ & $392(28.68)$ & & $30(32.57)$ & 156(29.88) & \\
\hline \multirow[t]{2}{*}{$\geq 75$} & $110(37.50)$ & $336(24.58)$ & & $23(27.71)$ & $108(26.42)$ & \\
\hline & $72.32 \pm 4.82$ & $70.01 \pm 4.58$ & & $72.32 \pm 4.82$ & $71.01 \pm 4.58$ & \\
\hline \multicolumn{7}{|l|}{ Residence, n (\%) } \\
\hline Rural & $38(11.26)$ & $553(36.77)$ & $<0.0001$ & $23(24.21)$ & 116(20.49) & 0.4852 \\
\hline Urban & 259(88.74) & $814(63.23)$ & & 72(75.79) & $359(79.51)$ & \\
\hline \multicolumn{7}{|l|}{ Education, $\mathrm{n}(\%)$} \\
\hline$\leq$ Elementary school graduation & $60(20.83)$ & $808(58.59)$ & $<0.0001$ & $51(59.08)$ & $255(55.05)$ & 0.7158 \\
\hline Middle school graduation & $30(9.85)$ & $207(15.52)$ & & $17(14.51)$ & $86(17.86)$ & \\
\hline$\geq$ High school graduation & 207(69.33) & $352(25.88)$ & & $27(26.41)$ & 134(27.08) & \\
\hline \multicolumn{7}{|l|}{ Partnered $^{\mathrm{d}}, \mathrm{n}(\%)$} \\
\hline Yes & $253(84.53)$ & 1037(74.25) & 0.0002 & $73(72.58)$ & $358(71.47)$ & 0.857 \\
\hline No & $44(15.47)$ & $330(25.75)$ & & $22(27.42)$ & 117(28.53) & \\
\hline \multicolumn{7}{|l|}{ Household income levele ${ }^{\mathrm{e}} \mathrm{n}(\%)$} \\
\hline Low or mid-low & $214(72.05)$ & 1174(85.88) & $<0.0001$ & $59(61.46)$ & $341(70.09)$ & 0.1637 \\
\hline Mid-upper or high & $83(27.95)$ & 193(14.12) & & $36(38.54)$ & 134(29.91) & \\
\hline \multicolumn{7}{|l|}{ Health-related behaviors } \\
\hline \multicolumn{7}{|c|}{ Body mass index $\left(\mathrm{kg} / \mathrm{m}^{2}\right), \mathrm{n}(\%)$ or mean $\pm \mathrm{SE}$} \\
\hline Underweight (<18.5) & $10(4.30)$ & $33(2.54)$ & 0.2624 & $4(4.60)$ & $10(2.16)$ & 0.3590 \\
\hline Normal (18.5-24.9) & $189(62.67)$ & $836(60.72)$ & & $55(59.95)$ & $275(57.54)$ & \\
\hline \multirow[t]{2}{*}{ Overweight and obese ( $\geq 25.0$ ) } & $98(33.03)$ & 498(36.74) & & $36(35.45)$ & 190(40.30) & \\
\hline & $24.05 \pm 2.89$ & $24.00 \pm 3.02$ & & $24.41 \pm 2.78$ & $24.16 \pm 3.01$ & \\
\hline \multicolumn{7}{|l|}{ Smoking status, n (\%) } \\
\hline Nonsmoker & $116(41.03)$ & $745(54.02)$ & $<0.0001$ & $10(10.53)$ & 238(52.12) & 0.8770 \\
\hline
\end{tabular}

a Propensity Score Matching.

${ }^{b}$ Retired status applies to individuals who were not working as they had reached the age of retirement and had already retired.

${ }^{c}$ Employed status applies to individuals who were working at least one hour per week for the purpose of income, or working 18 hours or more in a nonpaid family environment.

d Partnered refers to individuals who were residing with their spouses.

e Household income level was calculated by dividing the total household monthly income by the obtained levels and grouping the results into quartiles.

${ }^{f}$ Regular walking refers to having walked for more than 10 minutes a day for at least one day a week. 


\begin{tabular}{|c|c|c|c|c|c|c|}
\hline \multirow[t]{4}{*}{ Variable } & \multicolumn{3}{|c|}{ Before propensity score matching } & \multicolumn{3}{|c|}{ After propensity score matching } \\
\hline & Retired & Employed & P-value & Retired & Employed & P-value \\
\hline & Status ${ }^{b}$ & Status $^{c}$ & & Status & Status & \\
\hline & $(n=297)$ & $(n=1367)$ & & $(n=95)$ & $(n=475)$ & \\
\hline Ex-smoker & 153(51.21) & 459(33.37) & & $39(41.05)$ & 188(37.45) & \\
\hline Current smoker & $28(7.77)$ & $163(12.61)$ & & $46(48.42)$ & $49(10.43)$ & \\
\hline \multicolumn{7}{|l|}{ Alcohol consumption, n (\%) } \\
\hline Non-drinker & $99(34.91)$ & $578(42.04)$ & 0.0082 & $41(48.65)$ & 198(40.22) & 0.3052 \\
\hline$\leq 1 \mathrm{drink} /$ month & $63(18.94)$ & $306(21.55)$ & & $15(12.86)$ & $98(21.05)$ & \\
\hline 2 drinks/month to 3 drinks/week & $111(37.44)$ & $345(25.78)$ & & $31(29.21)$ & $126(27.43)$ & \\
\hline$\geq 4$ drinks/week & $24(8.71)$ & 138(10.63) & & $8(9.28)$ & $53(11.03)$ & \\
\hline \multicolumn{7}{|l|}{ Regular walking ${ }^{f}, \mathrm{n}(\%)$} \\
\hline No (or Rarely) & $39(14.55)$ & 405(29.03) & $<0.0001$ & $31(40.34)$ & $149(29.44)$ & 0.3052 \\
\hline Yes (or Regularly) & $258(85.45)$ & $959(70.97)$ & & $64(59.66)$ & $326(70.56)$ & \\
\hline \multicolumn{7}{|l|}{ Subjective health, n (\%) } \\
\hline Very good & $28(9.73)$ & $63(4.87)$ & $<0.0001$ & $7(11.36)$ & 22(11.36) & 0.0574 \\
\hline Good & $69(21.68)$ & $303(22.05)$ & & 18(16.36) & $96(16.36)$ & \\
\hline Moderate & 159(56.35) & 659(47.22) & & $52(52.44)$ & $237(52.44)$ & \\
\hline Poor & $41(12.24)$ & $342(25.86)$ & & 19(19.85) & 120(19.84) & \\
\hline \multicolumn{7}{|l|}{ a Propensity Score Matching. } \\
\hline \multicolumn{7}{|c|}{${ }^{\mathrm{b}}$ Retired status applies to individuals who were not working as they had reached the age of retirement and had already retired. } \\
\hline \multicolumn{7}{|c|}{$\begin{array}{l}{ }^{c} \text { Employed status applies to individuals who were working at least one hour per week for the purpose of income, or working } 18 \text { hours or more in a non- } \\
\text { paid family environment. }\end{array}$} \\
\hline \multicolumn{7}{|c|}{ d Partnered refers to individuals who were residing with their spouses. } \\
\hline \multicolumn{7}{|c|}{ e Household income level was calculated by dividing the total household monthly income by the obtained levels and grouping the results into quartiles. } \\
\hline
\end{tabular}


Table 2

Characteristics of the study population after propensity score matching (Gender disparity included)

\begin{tabular}{|c|c|c|c|c|c|c|}
\hline \multirow[t]{4}{*}{ Variable } & \multicolumn{3}{|l|}{ Men } & \multicolumn{3}{|l|}{ Women } \\
\hline & Retired & Employed & P-value & Retired & Employed & P-value \\
\hline & Status ${ }^{\mathrm{a}}$ & Status $^{b}$ & & Status & Status & \\
\hline & $(n=59)$ & $(n=292)$ & & $(n=36)$ & $(n=183)$ & \\
\hline \multicolumn{7}{|l|}{ Demographic factors } \\
\hline \multicolumn{7}{|l|}{ Age $(y), n(\%)$ or mean \pm SE } \\
\hline $65-69$ & $31(49.95)$ & 156(55.02) & 0.4898 & $11(26.76)$ & $55(27.32)$ & 0.9617 \\
\hline $70-74$ & 18(35.07) & $93(31.96)$ & & $12(29.41)$ & $63(26.88)$ & \\
\hline \multirow[t]{2}{*}{$\geq 75$} & $10(14.98)$ & $43(13.02)$ & & $13(43.83)$ & $65(45.80)$ & \\
\hline & $70.25 \pm 4.44$ & $70.12 \pm 4.04$ & & $72.72 \pm 5.39$ & $72.74 \pm 4.49$ & \\
\hline \multicolumn{7}{|l|}{ Residence, $\mathrm{n}(\%)$} \\
\hline Rural & $16(25.24)$ & $82(24.42)$ & 0.0171 & $7(23.26)$ & $34(14.81)$ & 0.3735 \\
\hline Urban & $43(74.75)$ & $210(75.58)$ & & $29(76.74)$ & 149(85.19) & \\
\hline \multicolumn{7}{|l|}{ Education, n (\%) } \\
\hline$\leq$ Elementary school graduation & $21(35.25)$ & 108(37.44) & 0.2949 & $30(85.44)$ & 147(80.53) & 0.7376 \\
\hline Middle school graduation & $16(24.77)$ & $78(28.20)$ & & $1(1.53)$ & $8(2.91)$ & \\
\hline$\geq$ High school graduation & $22(36.97)$ & 106(34.36) & & $5(13.03)$ & $28(16.56)$ & \\
\hline \multicolumn{7}{|l|}{ Partnered ${ }^{c}, \mathrm{n}(\%)$} \\
\hline Yes & $57(97.41)$ & $272(94.22)$ & 0.8657 & $16(41.14)$ & $86(38.58)$ & 0.8061 \\
\hline No & $2(2.59)$ & $20(5.78)$ & & $20(58.86)$ & $97(61.42)$ & \\
\hline \multicolumn{7}{|l|}{ Household income leveld ${ }^{d}$ n (\%) } \\
\hline Low or mid-low & $36(61.39)$ & 188(60.78) & 0.9351 & $23(61.54)$ & 153(83.56) & 0.0097 \\
\hline Mid-upper or high & $23(38.61)$ & 104(39.22) & & $13(38.46)$ & $30(16.44)$ & \\
\hline \multicolumn{7}{|l|}{ Health-related behaviors } \\
\hline \multicolumn{7}{|c|}{ Body mass index $\left(\mathrm{kg} / \mathrm{m}^{2}\right), \mathrm{n}(\%)$ or mean $\pm \mathrm{SE}$} \\
\hline Underweight $(<18.5)$ & $2(4.70)$ & $7(2.72)$ & 0.7643 & $2(4.48)$ & $3(1.36)$ & 0.1555 \\
\hline Normal (18.5-24.9) & $35(56.95)$ & 173(59.34) & & $20(63.75)$ & 102(54.93) & \\
\hline Overweight and obese $(\geq 25.0)$ & $22(38.35)$ & 112(37.94) & & 14(31.77) & $78(43.71)$ & \\
\hline \multicolumn{7}{|l|}{ Smoking status, n (\%) } \\
\hline Nonsmoker & $11(19.74)$ & $60(21.16)$ & 0.9159 & $35(98.03)$ & 178(96.91) & - \\
\hline Ex-smoker & $38(64.74)$ & 185(61.76) & & $1(1.97)$ & $3(2.29)$ & \\
\hline Current smoker & $10(15.52)$ & $47(17.08)$ & & $0(0.00)$ & $2(0.80)$ & \\
\hline \multicolumn{7}{|l|}{ Alcohol consumption, $\mathrm{n}(\%)$} \\
\hline Non-drinker & $15(26.65)$ & $79(22.61)$ & 0.5269 & $26(76.50)$ & 119(66.67) & 0.2881 \\
\hline$\leq 1 \mathrm{drink} /$ month & $9(13.34)$ & $52(18.44)$ & & $6(12.26)$ & $46(24.81)$ & \\
\hline
\end{tabular}

a Retired status applies to individuals who were not working as they had reached the age of retirement and had already retired.

${ }^{b}$ Employed status applies to individuals who were working at least one hour per week for the purpose of income, or working 18 hours or more in a nonpaid family environment.

${ }^{\mathrm{c}}$ Partnered refers to individuals who were residing with their spouses.

${ }^{\mathrm{d}}$ Household income level was calculated by dividing the total household monthly income by the obtained levels and grouping the results into quartiles.

${ }^{\text {e }}$ Regular walking refers to having walked for more than 10 minutes a day for at least one day a week. 


\begin{tabular}{|c|c|c|c|c|c|c|}
\hline \multirow{4}{*}{ Variable } & \multicolumn{3}{|l|}{ Men } & \multicolumn{3}{|l|}{ Women } \\
\hline & Retired & Employed & P-value & Retired & Employed & P-value \\
\hline & Status ${ }^{a}$ & Status $^{b}$ & & Status & Status & \\
\hline & $(n=59)$ & $(n=292)$ & & $(n=36)$ & $(n=183)$ & \\
\hline 2 drinks/month to 3 drinks/week & $28(47.68)$ & 112(41.14) & & $3(5.83)$ & $14(7.62)$ & \\
\hline$\geq 4$ drinks/week & $7(12.33)$ & $49(17.81)$ & & $1(5.41)$ & $4(1.90)$ & \\
\hline \multicolumn{7}{|l|}{ Regular walking ${ }^{\mathrm{e}}, \mathrm{n}(\%)$} \\
\hline No (or Rarely) & 16(31.94) & 77(22.77) & 0.1641 & 15(50.98) & $72(39.10)$ & 0.2845 \\
\hline Yes (or Regularly) & $43(68.06)$ & $215(77.23)$ & & $21(49.02)$ & $111(60.90)$ & \\
\hline \multicolumn{7}{|l|}{ Subjective health, n (\%) } \\
\hline Very good & $4(8.31)$ & 18(5.39) & 0.7720 & $3(15.22)$ & $4(1.76)$ & 0.0045 \\
\hline Good & 12(18.36) & $66(23.47)$ & & $5(13.84)$ & $30(15.39)$ & \\
\hline Moderate & $33(53.56)$ & $146(49.73)$ & & 19(51.02) & $91(49.91)$ & \\
\hline Poor & 10(19.77) & $62(21.41)$ & & $9(19.92)$ & $58(32.94)$ & \\
\hline \multicolumn{7}{|c|}{ a Retired status applies to individuals who were not working as they had reached the age of retirement and had already retired. } \\
\hline \multicolumn{7}{|c|}{$\begin{array}{l}\text { b Employed status applies to individuals who were working at least one hour per week for the purpose of income, or working } 18 \text { hours or more in a non- } \\
\text { paid family environment. }\end{array}$} \\
\hline \multicolumn{7}{|c|}{${ }^{\mathrm{c}}$ Partnered refers to individuals who were residing with their spouses. } \\
\hline \multicolumn{7}{|c|}{ d Household income level was calculated by dividing the total household monthly income by the obtained levels and grouping the results into quartiles. } \\
\hline
\end{tabular}

\section{Relationship Between Retired Status And Mental Health}

Overall, stress perception was low in the retired group, with men and women demonstrating similar trends in this regard. On the other hand, differences were observed for the incidence of depressive symptoms and the presence of suicidal ideation. Regarding the incidence of depressive symptoms, retired status was more influential for women than for men. We were not able to analyze the presence of suicidal ideation among men due to a lack of data; among women, the odds ratio (OR) was 6.39 (95\% Cl 0.64-64.48) in Model 2, which was adjusted for age and other variables. This indicates that after adjustment for other variables such as age, smoking, drinking, education level, household income, and physical activity, retired status significantly exerted a negative influence on the presence of suicidal ideation among women (Table 3). 
Table 3

Association between retirement status and mental health in Korean elderly $\geq 65$ years participating in KNHANES VI-VII (2013-2017)

Mental health

Unadjusted

Model $1^{b}$

Model $2^{c}$

OR $(95 \% \mathrm{Cl})^{\text {a }}$

P-value OR $(95 \% \mathrm{Cl})$

P-value

OR $(95 \% \mathrm{Cl})$

P-value

Total

Stress perception

Almost none

Low

1

\begin{tabular}{lll} 
& $<.0001$ & 1 \\
\hline $0.38-1.15$ & & 0.67 \\
\hline $0.25-1.62$ & & 0.64
\end{tabular}

$0.39-1.16$

$<0.0001$

1

$<.0001$

High

0.64

$0.25-1.62$

$<.0001$

1

2.99

$<0.0001$

1

$<.0001$

Yes

3.04

$0.61-15.06$

$2.99 \quad 0.60-15.00$

3.18

0.67-15.01

Suicidal ideation

\begin{tabular}{|c|c|c|c|c|c|c|c|c|c|}
\hline No & 1 & & $<.0001$ & 1 & & $<0.0001$ & 1 & & $<.0001$ \\
\hline Yes & 2.49 & $0.25-24.77$ & & 2.52 & $0.27-23.34$ & & 3.2 & $0.36-28.39$ & \\
\hline
\end{tabular}

Men

Stress perception

Almost none

1

$0.20-2.12$
$0.39-1.54$

$<.0001$

$<0.0001$

1

$<.0001$

Low

$\begin{array}{ll}0.77 & 0.20-2.12 \\ 0.65 & 0.39-1.54\end{array}$

$\begin{array}{ll}<.0001 & 1 \\ 0.80 \\ 0.66\end{array}$

0.40-1.58

0.79

$0.39-1.59$

High

$\mathrm{m}$ incidence

Depressive symptom incidence

\begin{tabular}{|c|c|c|c|c|c|c|c|c|c|}
\hline No & 1 & & $<.0001$ & 1 & & $<0.0001$ & 1 & & $<.0001$ \\
\hline Yes & 3.14 & $0.23-143.01$ & & 3.12 & $0.24-41.46$ & & 3.04 & $0.23-40.72$ & \\
\hline
\end{tabular}

Women

\section{Stress perception}

\begin{tabular}{llllllll} 
Almost none & 1 & & $<.0001$ & 1 & & $<0.0001$ & 1 \\
Low & 0.53 & $0.21-1.37$ & & 0.53 & $0.21-1.33$ & 0.64 & $0.25-1.65$ \\
\hline High & 0.58 & $0.15-2.25$ & 0.58 & $0.15-2.31$ & 0.56 & $0.14-2.31$
\end{tabular}

\section{Depressive symptom incidence}

\begin{tabular}{|c|c|c|c|c|c|c|c|c|c|}
\hline No & 1 & & $<.0001$ & 1 & & $<0.0001$ & 1 & & $<.0001$ \\
\hline Yes & 2.92 & $0.47-18.29$ & & 2.92 & $0.47-18.31$ & & 3.65 & $0.63-21.34$ & \\
\hline \multicolumn{10}{|c|}{ Suicidal ideation } \\
\hline No & 1 & & $<.0001$ & 1 & & $<0.0001$ & 1 & & $<.0001$ \\
\hline Yes & 2.98 & $0.25-35.22$ & & 2.98 & $0.24-36.47$ & & 6.39 & $0.64-64.38$ & \\
\hline
\end{tabular}

a OR = odds ratio; $95 \% \mathrm{Cl}=95 \%$ confidence interval.

${ }^{\mathrm{b}}$ Model 1 was adjusted by age.

${ }^{c}$ Model 2 was fully adjusted by age and other environmental factors such as smoking, alcohol consumption, education, household income, physical activity, duration of sleep, and comorbidities. 
Table 5

Influence of covariates of the association between retired status and mental health in part

\begin{tabular}{|c|c|c|c|c|c|c|c|c|c|c|}
\hline \multirow[t]{2}{*}{ Factors } & \multicolumn{5}{|l|}{ Total } & \multicolumn{5}{|l|}{ Men } \\
\hline & $\mathrm{B}^{\mathrm{b}}$ & se & $95 \% \mathrm{Cl}$ & & P-Value & $\mathrm{B}^{\mathrm{b}}$ & se & $95 \% \mathrm{Cl}$ & & P-Value \\
\hline Unadjusted & 0.035 & 0.031 & -0.026 & 0.096 & 0.257 & -0.234 & 0.280 & 0.455 & 1.376 & $<.0001$ \\
\hline $\begin{array}{l}\text { Age and } \\
\text { gender }\end{array}$ & 0.037 & 0.031 & -0.021 & 0.097 & 0.569 & -0.207 & 0.272 & 0.475 & 1.390 & $<.0001$ \\
\hline
\end{tabular}

\section{Somatic health ${ }^{\mathrm{a}}$}

\begin{tabular}{|c|c|c|c|c|c|c|c|c|c|c|}
\hline Hypertension ${ }^{a}$ & 0.037 & 0.031 & -0.035 & 0.097 & 0.705 & -0.236 & 0.278 & 0.471 & 1.412 & $<.0$ \\
\hline $\mathrm{OA}+\mathrm{RA}^{\mathrm{a}}$ & 0.036 & 0.030 & -0.024 & 0.096 & 0.321 & -0.200 & 0.268 & 0.483 & 1.388 & $<.0$ \\
\hline $\mathrm{DM}^{\mathrm{a}}$ & 0.037 & 0.030 & -0.023 & 0.097 & 0.153 & -0.212 & 0.275 & 0.471 & 1.390 & $<.0$ \\
\hline Osteoporosis ${ }^{a}$ & 0.036 & 0.031 & -0.024 & 0.097 & 0.676 & -0.208 & 0.271 & 0.476 & 1.388 & $<.0$ \\
\hline $\begin{array}{l}\text { All somatic } \\
\text { health }^{a}\end{array}$ & 0.036 & 0.030 & -0.023 & 0.095 & 0.534 & -0.210 & 0.277 & 0.47 & 1.398 & $<.0$ \\
\hline
\end{tabular}

Health-related

behavior $^{\mathrm{a}}$

\begin{tabular}{|lllllllllrr}
\hline Smoking $^{\mathrm{a}}$ & 0.037 & 0.031 & -0.024 & 0.097 & 0.728 & -0.207 & 0.273 & 0.475 & 1.392 & $<.0$ \\
\hline Drinking $^{\mathrm{a}}$ & 0.037 & 0.031 & -0.024 & 0.097 & 0.638 & -0.203 & 0.275 & 0.475 & 1.404 & $<.0$ \\
\hline BMl $^{\mathrm{a}}$ & 0.037 & 0.030 & -0.023 & 0.097 & 0.495 & -0.206 & 0.271 & 0.477 & 1.388 & $<.0$ \\
\hline Regular exercise $^{\mathrm{a}}$ & 0.038 & 0.031 & -0.023 & 0.098 & 0.432 & -0.207 & 0.273 & 0.475 & 1.391 & $<.0$ \\
\hline $\begin{array}{l}\text { All health } \\
\text { behavior }\end{array}$ & 0.038 & 0.030 & -0.022 & 0.097 & 0.600 & -0.201 & 0.274 & 0.476 & 1.404 & $<.0$ \\
\hline
\end{tabular}

Sociodemographic

factors ${ }^{a}$

\begin{tabular}{|c|c|c|c|c|c|c|c|c|c|c|}
\hline $\begin{array}{l}\text { Household } \\
\text { income }^{a}\end{array}$ & 0.037 & 0.031 & -0.023 & 0.097 & 0.399 & -0.210 & 0.270 & 0.476 & 1.381 & $<.0$ \\
\hline Education $^{a}$ & 0.037 & 0.031 & -0.024 & 0.097 & 0.729 & -0.212 & 0.271 & 0.474 & 1.382 & $<.0$ \\
\hline Partnered $^{\mathrm{a}}$ & 0.038 & 0.030 & -0.022 & 0.098 & 0.441 & -0.200 & 0.270 & 0.481 & 1.395 & $<.0$ \\
\hline $\begin{array}{l}\text { Urban-rural } \\
\text { gradient }^{a}\end{array}$ & 0.038 & 0.030 & -0.020 & 0.096 & 0.472 & -0.200 & 0.270 & 0.481 & 1.395 & $<.0$ \\
\hline $\begin{array}{l}\text { All } \\
\text { sociodemographic } \\
\text { factors }^{a}\end{array}$ & 0.040 & 0.029 & -0.018 & 0.097 & 0.313 & -0.204 & 0.267 & 0.482 & 1.382 & $<.0$ \\
\hline All covariates ${ }^{a}$ & & 0.028 & -0.015 & 0.095 & 0.250 & -0.205 & 0.272 & 0.476 & 1.394 & $<.0001$ \\
\hline
\end{tabular}

\section{Relationship Between Retired Status And Chronic Disease Incidence}

We examined the incidence of chronic diseases according to retired status, aiming to investigate whether retired status would influence other physical health aspects outside of mental health (Table 4). We calculated the OR values using regression analysis, and the results were very similar after adjusting for other chronic disease factors and demographic factors. In a chronic disease model adjusted for demographic factors and other chronic disease factors, retired status was correlated with high blood pressure $(\mathrm{OR}=0.91 ; 95 \% \mathrm{Cl}=0.51-1.60)$, osteoarthritis $(\mathrm{OR}=0.73 ; 95 \% \mathrm{Cl}=0.74-2.74)$, rheumatoid arthritis $(\mathrm{OR}=0.25$; $95 \% \mathrm{Cl}=0.03-2.44)$, diabetes $(\mathrm{OR}=0.50 ; 95 \% \mathrm{Cl}=0.26-0.95)$, osteoporosis $(\mathrm{OR}=0.78 ; 95 \% \mathrm{Cl}=0.36-1.68)$, and tuberculosis $(\mathrm{OR}=0.57 ; 95 \% \mathrm{Cl}=0.19-1.74)$. 
Table 4

Association between retirement status and chronic disease in Korean elderly $\geq 65$ years participating in KNHANES VI-VII (2013-2017)

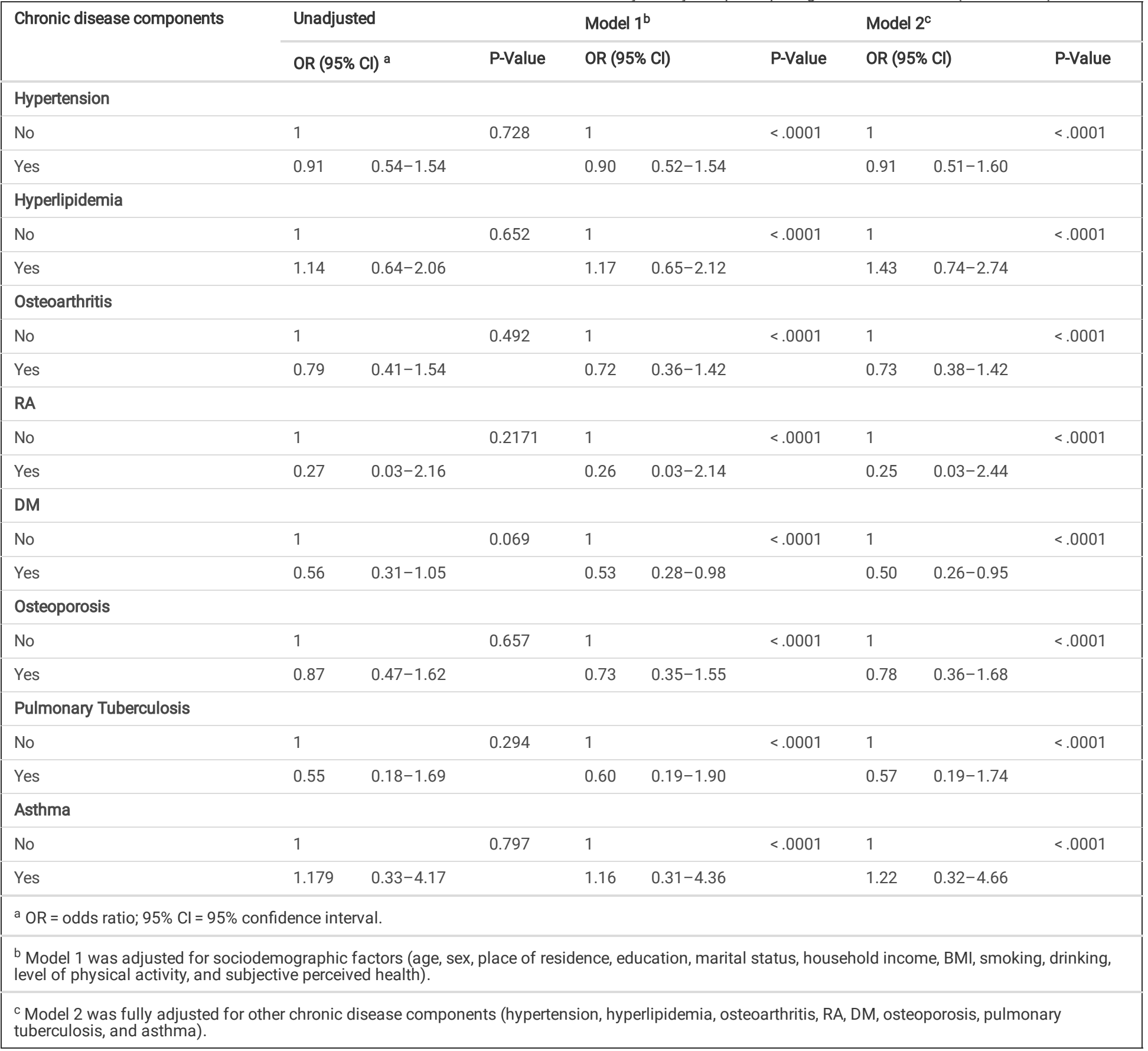

\section{Influence of covariates in the linear regression analysis on the correlation with retired status and mental health of elderly participants}

We evaluated the influence of each covariate block (somatic health, health related behavior, and sociodemographic factors) on the correlation between retired status and mental health after adjusting for age and sex (Table 5). The non-standardized regression coefficient relating to mental health among elderly men was -0.234 , which increased slightly after adjusting for age and sex; the non-standardized regression coefficient relating to mental health among elderly women decreased slightly to -0.309 after adjusting for age and sex. The negative values present for both men and women indicate that overall, retired status negatively influenced participants' mental health. Non-standardized regression coefficients were used as a standard of comparison with other variables. Therefore, adjusting for somatic health, health behavior, and sociodemographic factors, the absolute value of the difference between the two coefficients reflected the differences in effect size of each factor.

Among men, the absolute difference between -0.207 and the regression coefficients of each factor reflected the individual impact; the factor with the most significant influence was health-related behavior, which reduced the connection with mental health by $3 \%$. The variable with the most significant weakening influence among health-related behaviors was alcohol consumption (1.9\%). Sociodemographic factors weakened the association with mental health by $1.5 \%$; two notable individual factors were living with one's spouse and type of residence, with $3.4 \%$ in relevance. Among the physical health variables, only osteoarthritis and rheumatoid arthritis showed a weakening influence (3.5\%); the rest of the chronic disease variables did not have much impact. 
Using the same mechanism, we also explored factors that reduced the association with mental health among women; in terms of large categories, sociodemographic factors reduced the impact by $2.5 \%$, and the physical health and health-related behavior factors did not have much impact. However, we noted some differences in results when assessing the impact of individual factors. Osteoporosis, one of the sub-items within the physical health variable, indicated a relatively large impact size of $4.2 \%$. Among sociodemographic factors, household income level had a significant impact of a $22.8 \%$ reduction, whereas other factors were not very impactful.

When comparing results after adjusting for all variables, the association between retired status and mental health among the elderly decreased by $1 \%$ among men and increased by $28 \%$ among women. These results indicate that the mental health of men is more heavily impacted by retired status than the mental health of women.

\section{Discussion}

The mental health-related variables that were explored among the elderly in this study were divided into stress perception, psychiatric diagnosis of depression, and presence of suicidal ideation. Interestingly, they demonstrated different trends in being influenced by retired status. The observation that there were lower levels of stress perception among both men and women after retirement can be interpreted as the participants having previously been in stressful occupational and/or social environments that they no longer experienced. Furthermore, despite the lack of data as a limitation, retired status generally had a negative influence on the incidence of depressive symptoms and the presence of suicidal ideation.

The results of this study were not too far removed from those of the majority of previous studies with similar objectives. One such study found retirement to have a negative influence on mental health and morale [8], and another study reported that suicide rates were higher after retirement [9].

A major factor that impairs the mental health of the elderly post-retirement is retirement stress, which can be explained using the social stress model [10]. According to the model, retirement stress includes the feeling of loss due to reduced income, changes to daily life, deterioration of status both at home and in society, loss of self-identity, changes to one's social network, reduction of meaningful life experiences, and negative attitudes towards retirement [11].

Depression is one of the most common mental health symptoms that the elderly experience due to maladaptation to retirement stress. A study that analyzed the relationship between retirement and depression among the retired elderly found that retirement led to declines in mental health and expected that lower retirement satisfaction could lead to depressive symptoms [12]. Furthermore, another study reported that retired individuals experienced higher incidence rates of various mental illnesses, including depression, compared to those who are employed [13].

We found that changes to the physical and psychological environment due to retirement influence mental health and influence the occurrence of chronic diseases. Behncke et al. [14] utilized data from the English Longitudinal Study of Ageing to analyze the various effects of retirement on health results using non-parametric matching and tool variables (IV), concluding that retirement increases the risk of chronic condition diagnosis by $10 \%$. Particularly, they reported that retirement raises the risks of cardiovascular disease (4\%) and cancer (3-4\%) diagnoses, and that it was likely to cause issues in physical activity. Furthermore, a study by Alvinia found that leaving the labor market has a negative influence on chronic disease occurrence in many European countries [15].

In addition to yielding cross-sectional results, this study aimed to discover the most influential factors among multiple covariates that were impactful on the influence of retired status on elderly mental health. Among numerous results that can be found in Table 5, some notable points included that alcohol consumption was the most influential covariate among men, and that the sociodemographic block was the most influential among women.

Drinking in old age can lead to various geriatric physical conditions, and often causes psychiatric issues [16]. Experiencing a loss of social roles, reduced economic power, loneliness and alienation, and deaths of spouses after retirement leads to the experience of feelings such as helplessness, isolation, and selfblame; this may lead to new tendencies to drink or binge drink, or exacerbate existing problematic drinking [17]. Particularly, middle-aged men in Korean society may experience excessive stress and maladaptation to their post-retirement lives and are likely to engage in problematic drinking [18].

On the other hand, it is important to compare the retirement environment that women face when discussing the issue of retirement for elderly Korean women. Female Korean retirees comprise a generation that had to construct their own career paths while facing a lack of understanding regarding women's employment due to the residue of patriarchal values; as such, they maintained their occupational status amidst social expectations and norms that differed from those for men, who were praised as work-oriented tendencies were equated with the responsibilities of the breadwinner [19]. Considering the particular cultural environment that Korean employed women faced, we are able to infer that the influence of sociodemographic factors will be significant.

There are two major points of views on elderly retirement. One point of view is viewing retirement as a crisis. In other words, retirement is regarded as a significant crisis faced during the later half of one's life, resulting in a loss of status, a loss of self-identity, non-productive leisure time, and the social stigma of incompetence. On the other hand, the other point of view considers retirement to be a continuous phase of the life cycle. This viewpoint regards retirement as a predictable process, rather than a crisis that one abruptly faces. Furthermore, this viewpoint does not equate work with life, but instead regards it as a temporary task at a specific stage of life, asserting that retirement is not as significant a crisis as is commonly believed. Reviewing these two contrasting viewpoints in relation to the cultural characteristics of Korean society and mental health of the elderly, we are able to conclude that in Korea, retirement is likely to be perceived as a stressful event for the individual instead of as a simple change in environment. This is due to factors such as a comparatively younger retirement age, a lack of preparation for retirement, involuntary retirement, and weak social safety nets; furthermore, it can also be predicted that postretirement adaptation will be relatively difficult [20].

However, not everyone who retires experiences declines in mental health. Jokela et al. [21] asserts that retirement has a positive influence on mental health, and that legal retirement and early voluntary retirement improve health status and physical functions. In a similar context, Charles et al. [22] found that freedom from labor via retirement has a positive influence on life satisfaction.

Page $12 / 15$ 
These differences can be attributed to individual vulnerabilities; although the retirees experience stress from retirement, an environmental event, the possibility of experiencing deteriorated mental health changes depending on the individual's level of vulnerability. This can be explained using the stress-vulnerability model. The stress-vulnerability model is useful in understanding the causes and processes inducing mental illnesses through the interaction between individual vulnerability and environmental stress [23], and existing studies $[24,25]$ have attempted to approach elderly mental health issues through this model.

The main advantages of the present study are as follows. First, the health-related survey used in this study was conducted by trained professionals using a national sample of Koreans in a systematic manner. This study is valuable as it is the first study to comprehensively evaluate the effect of retirement on the mental health status of elderly Koreans on a national level. Another advantage is that we went beyond exploring cross-sectional association by adjusting factors that could act as confounding variables in the association between retired status and mental health variables to explore the level of influence of such factors on retirement and mental health levels.

However, this study also has the following limitations. First, given the cross-sectional study design, which limits the study to a short and specific period of time, this study can only derive associations rather than causal relationships.

Another limitation of this study is that the response data reflecting the mental health status of the elderly, such as levels of perceived stress and presence of suicidal ideation, were self-reported. This is a limitation because self-report surveys can lead to subjective and biased results, which differ from physicians' diagnoses or diagnostic testing results. Furthermore, these self-report surveys carry the risk of being exposed to memory bias.

Lastly, a clear limitation of this study is the failure to obtain sufficient data for certain factors, which may have led to these factor acting as confounding variables in the regression analysis despite the fact that this study was carried out based on the results of a national survey. First, when analyzing healthrelated behaviors, we were not able to reflect the elderly female smoker population as there were no smokers among the studied female retirees. Furthermore, when examining the presence of suicidal ideation as one of the mental health variables, we were not able to examine this factor among men due to a small sample size. Although we were able to obtain an OR value of 3.2 across both genders, it would be difficult to compare gender-specific differences for this factor.

\section{Conclusions}

Ultimately, the experience of retirement in old age is negatively associated with the mental health of the elderly and health-related behaviors such as drinking and sociodemographic factors strengthen this negative relationship. These results were more pronounced among women, perhaps due to the characteristics of Korean culture that reflect different job perceptions and gender roles for women and men. Follow-up studies should identify the mechanisms underlying declines in mental health over time during retirement and explore protective factors that can mediate the association between the continuation of retirement and the deterioration of mental health.

\section{Abbreviations}

$\mathrm{Cl}$, confidence interval; KCDC, Korea Center for Disease Control and Prevention; KNHANES, Korean National Health and Nutrition Examination Surveys; OR, odds ratio; PSM, propensity score matching; DM, diabetes mellitus; RA, rheumatoid arthritis.

\section{Declarations}

\section{Ethics approval and consent to participate}

IRB approval was not required for the present study because it did not involve any sensitive information and accessed only publicly available data from KNHANES (JASENG IRB File No. 2020-03-002).

\section{Consent for publication}

Not applicable.

\section{Availability of data and materials}

The datasets generated and analysed during the current study are available in KNHANES repository (http://knhanes.cdc.go.kr). All data from KNHANES VI-VII are coded and freely available.

\section{Competing interests}

The authors declare that they have no competing interests.

\section{Funding}

This research was funded by the Jaseng Medical Foundation, Republic of Korea.

\section{Authors contributions}


TYK analysed and interpreted the data and drafted the manuscript. SHL acquired, analysed and interpreted the data. IHH acquired the data and made substantial contributions to the conception and design of the study. EJK, JHK, and SYC revised the draft manuscript. All authors read and approved the final manuscript.

\section{Acknowledgements}

Not applicable.

\section{References}

1. Elliott MS, Barris R. Occupational role performance and life satisfaction in elderly persons. Occup Ther J Res. 1987;7:215-24.

2. Westerlund H, Vahtera J, Ferrie JE, Singh-Manoux A, Pentti J, Melchior M, et al. Effect of retirement on major chronic conditions and fatigue: French GAZEL occupational cohort study. BMJ. 2010;341:c6149.

3. Bromley DB. Behavioural gerontology: central issues in the psychology of ageing.: John Wiley \& Sons, Inc.;1990.

4. Dorfman LT, Moffett MM. Retirement satisfaction in married and widowed rural women. Gerontologist. 1987;27:215-21.

5. $10.3386 / \mathrm{w} 12123$

Dave D, Rashad I, Spasojevic J. The effects of retirement on physical and mental health outcomes. No. w12123. National Bureau of Economic Research. 2006. https://doi.org/10.3386/w12123. Accessed.

6. Butterworth P, et al. Retirement and mental health: analysis of the Australian national survey of mental health and well-being. Soc Sci Med. 2006;62:1179-91.

7. Song J, et al. The effects of marital status and retirement status on mental health among older adults: a longitudinal U.S. national study. Korean J Popul Stud. 2007;30:25-47.

8. Maddox GL. Sociology of later life. Annu Rev Sociol. 1979;5:113-35.

9. Bossé R, et al. How stressful is retirement? Findings from the Normative Aging Study. J Gerontol. 1991;46:9-14.

10. The psychology of retirement:;1996.

Bossé R, Spiro A III, Kressin NR. The psychology of retirement.;1996.

11. George LK. Role transitions in later life.:Brooks/Cole Pub. Co.;1980.

12. Higginbottom SF, Julian B, Kelloway EK. Linking retirement experiences and marital satisfaction: a mediational model. Psychol Aging. 1993;8:508.

13. Gill SC, et al. Mental health and the timing of men's retirement. Social Psychiatry Psychiat Epidemiol. 2006;41:515-22.

14. Behncke S. Does retirement trigger ill health? Health Econ. 2012;21:282-300.

15. Alavinia SM, Burdorf A. Unemployment and retirement and ill-health: a cross-sectional analysis across European countries. Int Arch Occ Env Hea. 2008;82:39-45.

16. Perreira KM, Sloan FA. Excess alcohol consumption and health outcomes: a 6-year follow-up of men over age 50 from the health and retirement study. Addiction. 2002;97:301-10.

17. Brennan PL, Schutte KK, Moos RH. Retired status and older adults' 10-year drinking trajectories. J Stud Alcohol Drugs. 2010;71:165-68.

18. Kim KW, et al. Prevalence and neuropsychiatric comorbidities of alcohol use disorders in an elderly Korean population. Int J Geriatr Psych. 2009;24:142028.

19. Lee J, Kim M-H. The effect of employment transitions on physical health among the elderly in South Korea: a longitudinal analysis of the Korean Retirement and Income Study. Soc Sci Med. 2017;181:122-30.

20. Easterlin RA, MacDonald C, Macunovich DJ. Retirement prospects of the baby boom generation: A different perspective. Gerontologist. 1990;30:776-83.

21. Jokela M, et al. From midlife to early old age: health trajectories associated with retirement. Epidemiology. 2010;21:284.

22. Charles KK. Is retirement depressing? Labor force inactivity and psychological well-being in later life. Res Labor E. 2004;23:269-99.

23. Kahana E, et al. The effects of stress, vulnerability, and appraisals on the psychological well-being of the elderly. Res Aging. 1995;17:459-89.

24. Shields CG, Wynne LC. The strength-vulnerability model of mental health and illness in the elderly.;1997.

25. Oldehinkel AJ, et al. Psychosocial and vascular risk factors of depression in later life. J Affect Disorders. 2003;74:237-46.

\section{Figures}




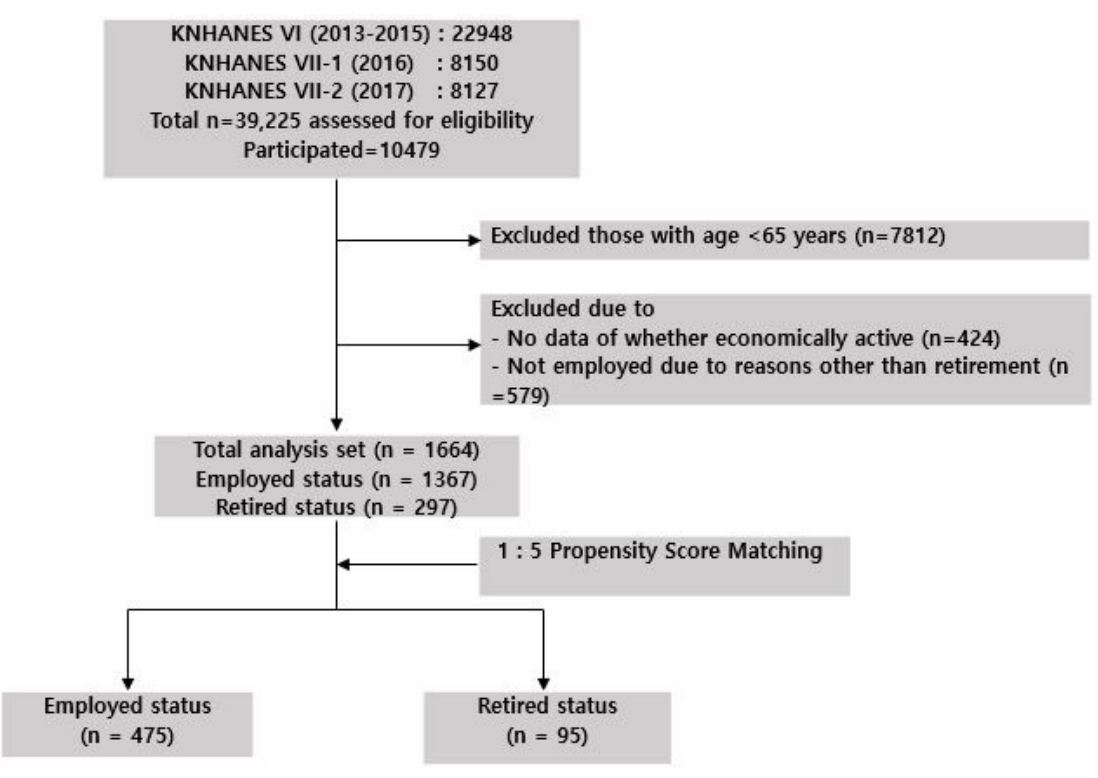

Figure 1

Flow diagram of inclusion and exclusion of respondents from KNHANES VI-VII (2013-2017).

\section{Supplementary Files}

This is a list of supplementary files associated with this preprint. Click to download.

- CertificateofeditingJASME402.pdf 